\title{
Experimental Studies of Conductive Paths of Printed Circuit Boards by Using Subminiature Eddy Current Transducers
}

\author{
Vladimir Malikov ${ }^{1, *}$, Maxim Ananyev ${ }^{2}$, Alexey Ishkov² and Leonid Nikonov ${ }^{3}$ \\ ${ }^{1}$ Altay State University, Lenina, 61, Barnaul, Russia \\ ${ }^{2}$ Altay State Agricultural University, Krasnoarmeysky, 73, Barnaul, Russia \\ ${ }^{3}$ Ankara Yildirim Beyazit University, Dumlupinar, 06760 Çubuk/Ankara, Turkey
}

\begin{abstract}
This paper describes the development and experimental study of a software and hardware complex designed for studying the conductive tracks of printed circuit boards. In the course of this development, a subminiature eddy-current transducer and a measuring system capable of performing measurements using the newly developed transducer were created. The paper contains descriptions of the main parameters of the developed measuring system, and also presents the results of experiments conducted with various printed circuit boards, both with defect-free and those with artificial model defects. These experiments made it possible to confirm the possibility of using the developed measuring system and eddycurrent transducer for controlling the conductive tracks on printed circuit boards and determining their quality.
\end{abstract}

\section{Introduction}

One of the important both fundamental and applied problems of physical material science is the development and improvement of research methods and diagnostics of the physicochemical and operational characteristics of materials used in modern industry and research and development. The production and operation of a number of high-tech products used in the aerospace industry, transport sector, nuclear industry and under extreme conditions of mining is impossible without their quality control.

Control and diagnostics are the starting and defining elements of the security problem. Control means the conformance checking of the object parameters with the established requirement specifications.

Thereat, non-destructive quality control methods become the most relevant, as they are the only ones which make it possible to analyze objects without interfering with their internal structure and changing their operational properties, thus allowing to carry out a complete quality check directly while in operation, if necessary.

Electronics is one of the fastest evolving, most innovative, and most competitive industries [1]. The past five years, have been characterized by growth in emerging markets and introduction of new products, leading more people to buy consumer electronics. The

* Corresponding author: osys11@gmail.com 
global consumer electronics industry was valued at \$1 trillion in 2020 [2]. Production of electronics involves many manufacturing processes from automated assembly lines to testing and final manual assembly [3]. The electronics product sizes are getting larger for home display devices such as the LCD TV sets. Or, the product sizes are dramatically reduced for handheld or wearable devices such as smart phones and smart watches, as a result the need for an AOI system to the various sizes of electronics products were not an exception from any other industry [4]. In fact, the challenges facing the electronics industry is much severe compared to others, as the electronics products and manufacturing plans evolve rapidly and the electronics products tend to have short life cycles [5]. These challenges require to change the process parameters accordingly. All of these changes (whether they are sudden or longterm) cause defective products, and therefore quality assurance and process improvement is very essential in electronics industry [6].

One of the main building blocks of modern electronics is considered the printed circuit board (PCB) which mechanically supports and electrically connects electronic components using conductive tracks, pads and other features etched from copper sheets laminated onto a non-conductive substrate. However, operation and even technology cause mechanical interactions between PCB and electronic components through the whole range of interlaying linking parts. Such interaction causes strain and stress as in bodies of the components so in contact joints what often causes their damage and failures of the whole electronic unit and therefore requires detailed studying. The general technology for components installation (e.g. capacitors, resistors or active devices) on PCBs remains soldering [7]. The soldered joint (SJ) strength is one of the indicators applied to assess its quality. Thus, developing methods for testing strength of soldered joints in PCBs is the objective for the current research.

As is well known, the reliability of printed circuit boards (PCBs) has a direct impact on the profitability of the entire production of radio-electronic equipment. Despite the strict adherence to etching processes and careful selection of materials, chemical processes used in etching still sometimes cause defects in the PCB (rupture, conductor short-circuiting, cracks, etc.).

Currently, it is a very topical task to identify dangerous technological defects during the production stages in order to limit release to service of potentially unreliable equipment. Modern methods of defect detection make it possible to find and localize most of the obvious and some of the hidden defects in radio-electronic equipment (REE).

Timely detection of such hidden defects and malfunctions as the microcracks, microroughnesses, and microgaps formation in communication lines and connectors, as well as aging or failure of printed circuit board elements during operation is one of the conditions for the reliability assurance of electronic equipment.

In the production of PCBs in the electronics industry, the substrate made of dielectric holds the various electronic components together.

The large concentration of probes on the boards causes a flexure of the boards due to the applied stress. This could result in solder ball cracking, pad lifting and damage to the traces on the board. After the assembly of components on the PCB substrate, the boards are tested for faults. The testing is usually done using an in-circuit test fixture [8]. In literature, after testing, scooping detection of solder paste using visual inspection of PCBs has been done [9]. Strain and fatigue in surface mount solder joints in PCBs were monitored in [10] using a destructive method. Sensors made of optical fibers and piezoelectric materials have been used for structural health monitoring These sensors have to be implanted inside the structure for in-situ monitoring. Also optical fiber arrays have been used to examine cracks and delamination under a bonded structure [11]. However, the use of implanted optical fibers brings down the strength of these materials while the replacement and maintenance of the fiber is very difficult and costly [12]. Also eddy current techniques for structural health 
monitoring have been employed to examine Strain and fatigue in surface mount solder joints in PCBs [13].

The ability to determine that a part is on the brink of failure, or beginning to show the precursors to failure, is valuable information. When used properly, this information can be used to realize increased aircraft availability, decreased maintenance costs and reduced spares costs. For electronic systems, however, the current state-of-the-art has not yet been able to determine reliably and repeatably when a circuit board is starting to exhibit signs of impending failure. The failure effects of integrated circuits are understood. These include such phenomena as metal migration, gate oxide breakdown, voids, solder joint breakdown, etc. However, the interdependency of these failure effects and the associated failure modes that affect the normal operation of a circuit are not well understood. Currently, complex and expensive laboratory test systems exist to identify specific failure modes through acoustic imaging and analysis. These systems utilize Scanning Acoustic Microscopy (SAM), Laser Doppler Vibrometry, or a combination of multi-spectral techniques to produce images of circuits and chips for failure mode and fault analysis [14-19]. Other techniques involve measuring the acoustic emissions from electronics while the system-under-test is undergoing mechanical stress [7]. Although acoustic imaging techniques have a proven track record in non-destructive testing (NDT), only a few published works in this area leverage nonimaging approaches to NDT, such as acoustical resonance processing and direct analysis of circuit components for failure conditions [20]. Although acoustical resonance analysis and characterization has not been applied specifically to circuit card testing, these techniques have a strong presence in failure detection of larger mechanical systems [21].

ECT is a well known method of NDT, usually applied to evaluate material flaws without changing or altering the same conducting materials. GMR sensors have recently applied to the material inspection in order to detect material cracks, based on the ECT and providing good inspection results [22]. ECs are induced in a specimen as a result of the application of an alternating magnetic field. For instance, a cylindrical coil could be used to produce an exciting magnetic field able to induce circular ECs constrained in an area nearby the specimen's surface. In presence of a defect, ECs' flow is perturbed: this phenomenon can be also quantified as a variation of the impedance in the exciting coil. Within this framework, GMR sensors have high sensitivity over a large frequency range and a high spatial resolution because their small dimensions, thus providing a good trade-off in terms of performance versus economic cost. It has been demonstrated that the magneto-resistive probes perform better than conventional probes for low-frequency applications, i.e., when detecting deep buried flaws. This is because the electromagnetic sensors are sensitive to the magnitude of the magnetic field [23].

Thus, the purpose of this study was to develop and investigate a new type of eddy-current measuring systems allowing to perform qualitative studies of conductive tracks of printed circuit boards, providing the ability to find small defects in these tracks and perform studies of closely spaced tracks of printed circuit boards.

\section{Experimental technique}

A scanning system includes a surface eddy-current transducer (ECT) consisting of three elements: a coaxial generating inductance coil, a measuring coil, and a special circuit necessary for compensation of the generating coil effects on the readings $[24,25]$. The general scheme of system operation is as follows: the generator generates current that is directed to the transformer generating winding and produces a magnetic field. The measuring and compensation loops are included in the circuit in such a way that the ECT signal would be equal to zero in the absence of an object under control near the transformer. When a conducting object appears in the coverage area of the measuring winding, the magnetic field 
from this coil induces eddy currents in the object under control. In turn, these eddy currents initiate the appearance of a useful signal about the structure of the substance in the measuring coil.

In addition to the above mentioned, the system includes an additional electronic unit. Its functional load consists in the quantitative indication of the signal recorded from the measuring coil. Thereat, the received signal represents a change in the electromotive force induced on the measuring circuit of the transducer.

Within the scope of this work, system's ECT has the following parameters: the diameter of the generating winding is $0.5 \mathrm{~mm}$, and that of the measuring winding is $0.1 \mathrm{~mm}$. The frequency of the current applied to the generating circuit was selected in such a way as to preserve the sensitivity of the system at depths of up to $0.5 \mathrm{~mm}$ and was $500 \mathrm{~Hz}-10 \mathrm{kHz}$. The technological gap between the surface of the object under control and the transducer was $0.2 \mathrm{~mm}$.

The described ECT is under the control of the developed VDSS-8 hardware and software complex. In order to prepare the readout indicators for visualization, the signals received from the transducer were processed by the electronic unit of the measuring transformer combined with the spectrum analyzer of the recorded signal. As a result, it became possible to display a picture on the screen and visually present the received data in a form convenient for perception. The hardware and software complex was designed in such a way as to provide the ability to fine-tune the scanning system for a specific control object, thus increasing the efficiency and convenience of system operation. The adjustment of the system supposes entering the amplitude and phase parameters into the computing unit followed by determination of the inverse transform coefficient. The internal system data is adjusted in accordance with the thickness of the control object and the technological gap between the object and the transducer. At the next step, the signal indicators are recorded in the absence of a control object and with a zero gap between it and the transducer. These data play the role of extreme beacons, between which the true values of the subsequent measurements will be located.

Upon completion of the adjustment step, the transducer is positioned over the object surface in such a way as to enable moving the ECT at a constant speed and transmit the received data in the form of a curve referenced to the points of object's welded seam. The coordinate of the ECT location was changed discretely in increments of $0.5 \mathrm{~mm}$ and was taken as the distance from the edge of the object to the point of the current location of the transducer at a given moment. During measurement, the value of the voltage introduced into the measuring winding was chosen as the parameter carrying the useful information load.

\section{Research results and their discussion}

Experiments on the defect-free conductive tracks of printed circuit boards were performed on Dell motherboard. Four conducting tracks (Fig. 1) of different widths (from 3.3 to 0.7 $\mathrm{mm})$, located at different distances from each other $(0.8$ and $0.6 \mathrm{~mm})$, were used as the object of research; scanning was carried out in the direction indicated by the arrow.

In the study, the eddy-current transducer exciting winding voltage was $2 \mathrm{~V}$.

Data on tracks studying using various designs of eddy-current transducer are shown in Fig. 2. Good contrast may be seen between the eddy-current transducer designs with a conventional ferrite core (Fig. 2.b) and an annealed permalloy-based core (Fig. 2.a.) The transducer on the permalloy-based core annealed at a special thermal pattern has detected all four conductive tracks, regardless of their width. The ferrite core detected only one track out of four. 


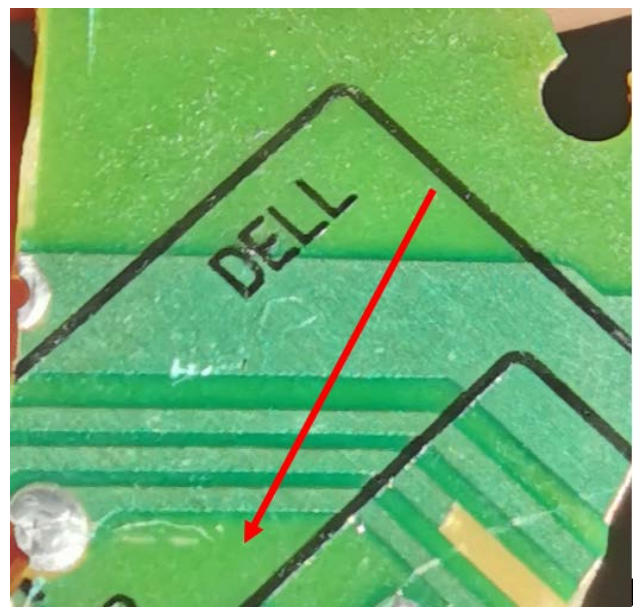

Fig. 1. Defect-free tracks 3.3 and $0.7 \mathrm{~mm}$ wide located at a distance of $0.8 \mathrm{~mm}$ and $0.6 \mathrm{~mm}$.

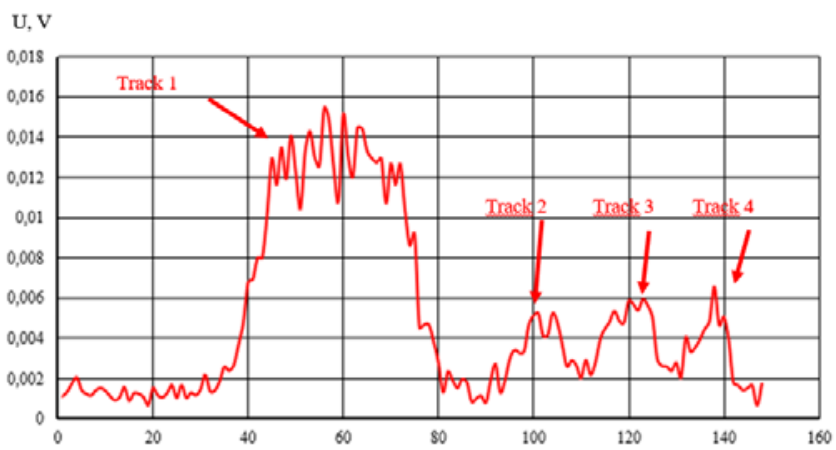

a)

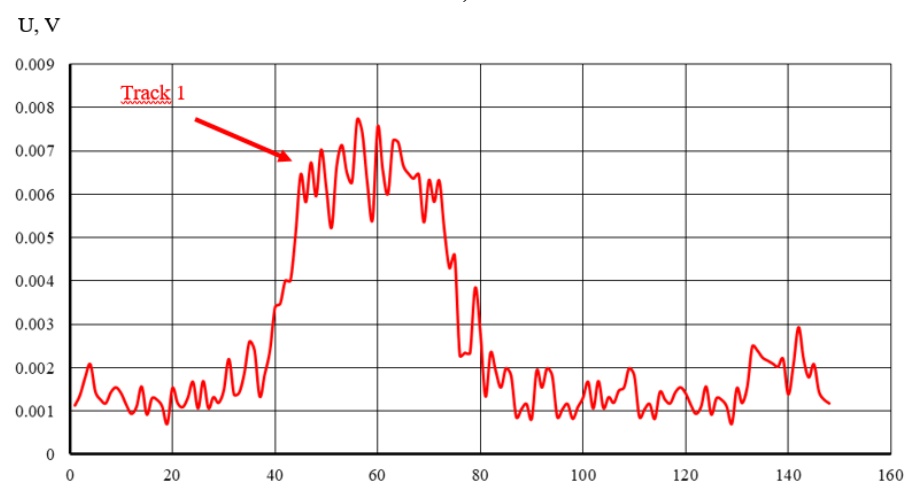

b)

Fig. 2. System scanning results of the conductive tracks using a measuring system with ferrite (a) and 81HMA permalloy-based (b) ECT, U - measuring winding voltage

\section{Investigation of defective conductive paths of printed circuit boards}

Experiments on studying of defect-free conductive paths of printed circuit boards were carried out on a Dell motherboard. As a research object, a conductive track with a model defect (Fig. 3) was used located separately from other tracks so that the signal from other 
tracks did not affect the ECT signal received from the main track. Scanning was performed at a frequency of $5000 \mathrm{~Hz}$.

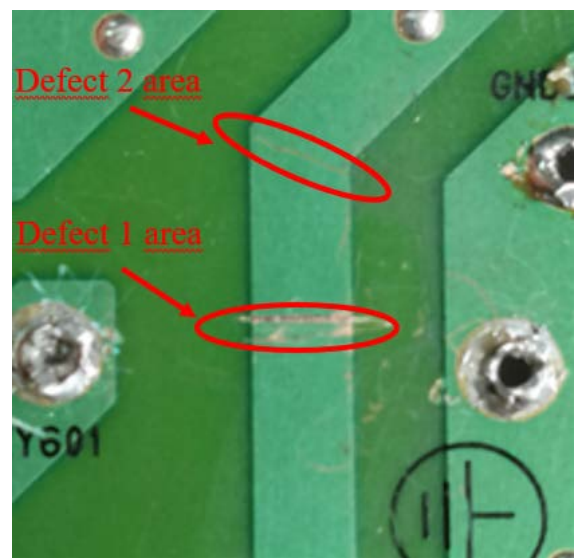

Fig. 3. Defective track $4.6 \mathrm{~mm}$ wide, defect 1 width is $0.3 \mathrm{~mm}$ defect 2 width is $0.05 \mathrm{~mm}$.

The results of experiments conducted on the board with a defective track are shown in Fig. 4. The presented dependences clearly show the difference in signals from two types of eddy-current transducers. The signal from the transducer based on annealed permalloy (Fig. 4.b) provides a much more pronounced picture of the defect in comparison with the signal from the ferrite-based transducer (Fig. 4.a). The defects in Fig. 4.b are clearly visible distinguishable by a significant drop of the ECT measuring winding signal voltage.

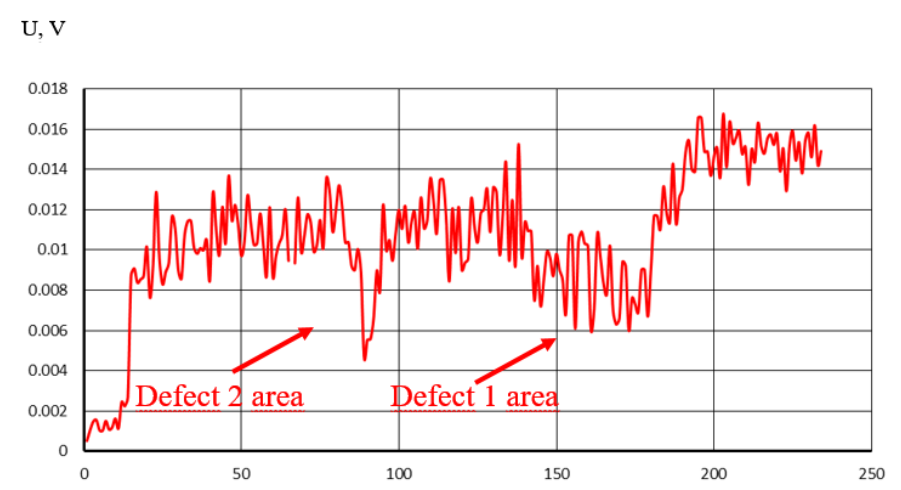

a)

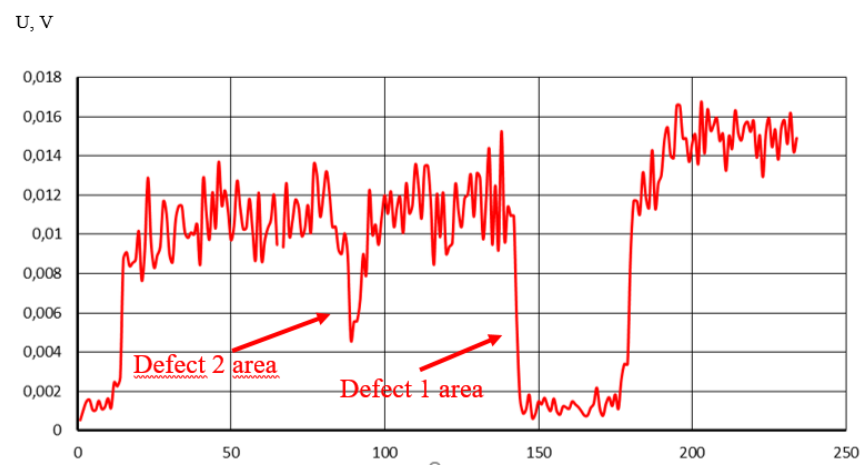

b)

Fig. 4. Results of scanning a conductive tracks system using a measuring system with a ferrite-based (a) and a 81NMA permalloy-based (b) ECT, U is the measuring winding voltage 
Investigation of the influence of the core shape on the signal of eddy-current transducer from various model defects

A number of cores in various shapes were used in the work. Figure 5 shows a trapezoidal core. Figure 6 shows a core in the form of a pointed cone. The cores were made of $2000 \mathrm{NMZ}$ ferrite with an initial permeability of 500, and 81NMA alloy with an initial permeability of 35000 .

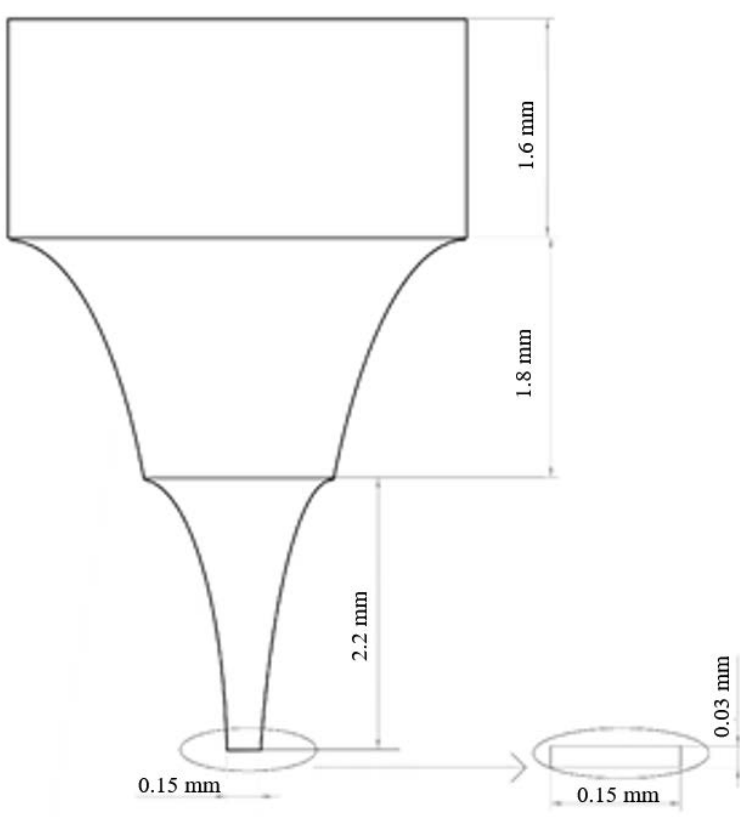

Fig. 5.a. ECT core No. 1

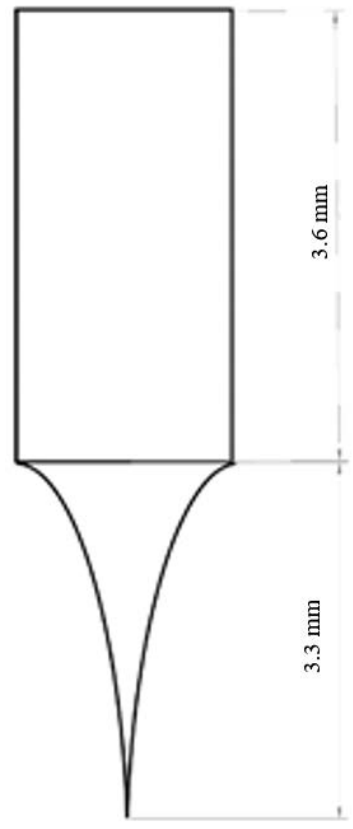

Fig.5.b. ECT core No. 2

On the basis of the cores used, a series of eddy-current transducers have been designed with exciting, measuring and compensation windings. The compensation winding is connected to the measuring winding and is intended for subtracting the exciting winding signal from the resulting one. The exciting and measuring windings contain 200 turns. Copper wire $5 \mu \mathrm{m}$ thick is used to wind the turns. The compensation winding contains $160 \div$ 180 turns.

The measuring winding diameter of ECT-1 was $0.15 \mathrm{~mm}$. The excitation winding had a diameter of $0.3 \mathrm{~mm}$. In ECT-1, a trapezoidal core was used. The dimensions of the measuring winding of ECT-2 were $0.1 \div 0.15 \mathrm{~mm}$. The exciting winding had a diameter of $0.5 \mathrm{~mm}$. In ECT-2, a pointed cone core was used.

The results of flaw detection using an ECT based on core No. 1 demonstrated the possibility of a more precise localization of the defect, as the signal did not drop too sharply when approaching the defect (Fig. 6), in comparison with the results of core No. 2 (Fig. 7 ), which may be explained by a more integral nature of the signal from the trapezoidal core. However, with the first type of core, defect No. 2 was missed, it became indistinguishable against the noise background. In the case of core No. 2, the localization of defect No. 1 was not so obvious, however, defect No. 2 was clearly distinguishable. 


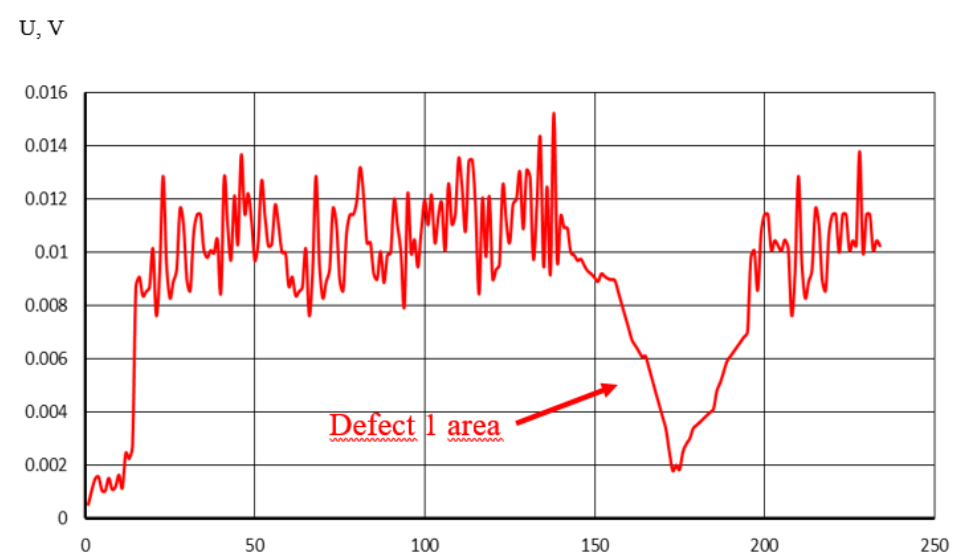

Fig. 6. Scanning results for the defective track of printed circuit board using core No. 1

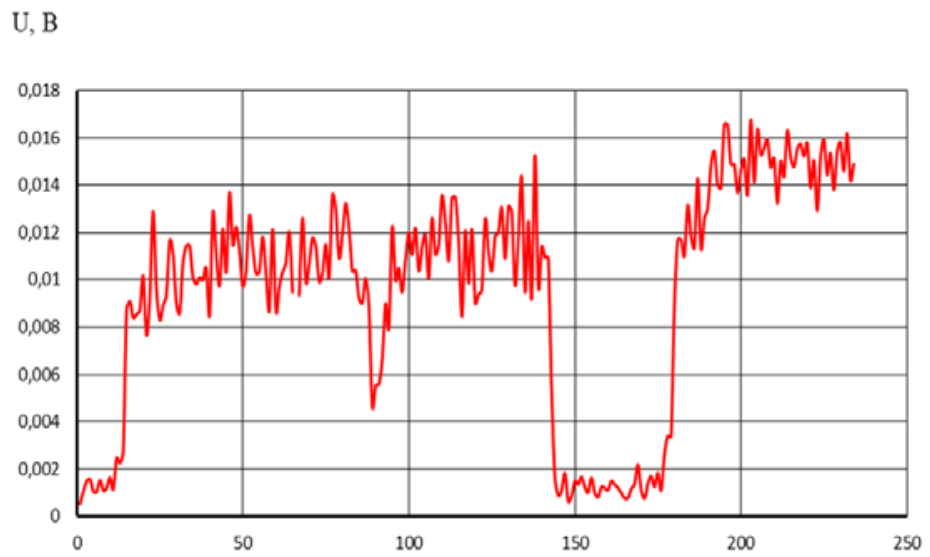

Fig. 7. Scanning results for the defective track of printed circuit board using core No. 2

\section{Defect scanning in closely spaced PCB tracks}

To scan closely spaced conducting tracks, it is necessary to select carefully the frequency of a signal applied to the exciting winding of the eddy-current transducer in order to achieve the largest measuring winding signal value of the eddy-current transducer. Therefore, calibration of the transducer was performed before starting the measurements consisted in determining the applied voltage from the defect-free area. The calibration was carried out at a frequency of $10,000 \mathrm{~Hz}$. After that, the transducer was passed over the scanned defective area. At that, the frequency was varied in the range of $100-1000 \mathrm{~Hz}$ with an increment of $100 \mathrm{~Hz}$. The frequency was determined providing the greatest deviation of the voltage introduced into the measuring winding from the voltage value obtained in the defect-free area of the specimen. The voltage value corresponding to this frequency was considered as a parameter the behavior of which was used to judge the presence of a defect.

The diameter of the transducer measuring winding used for scanning was $0.5 \mathrm{~mm}$, the size of the scanning area on the specimen surface was $0.1 \mathrm{~mm}$, the time per measurement at one frequency was $0.1 \mathrm{~s}$, and 1 second when all frequencies were scanned, the calibration time was 0.5 second.

In the course of this study, the task was to search for a defect on a conductive track of a printed circuit board, provided that another track is located near the track under study, making 
a significant contribution to the ECT voltage (Fig. 8). The experimental results are shown in Fig. 9.

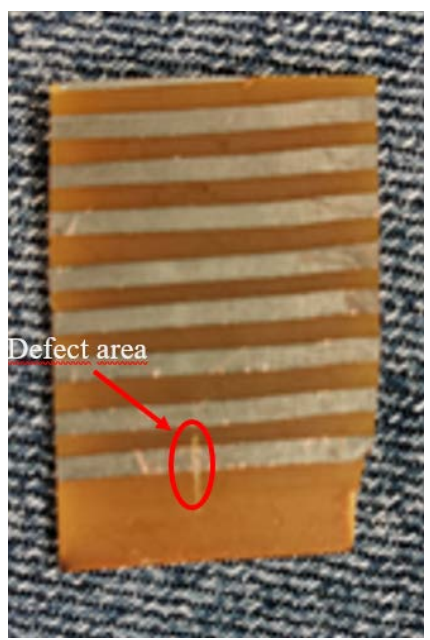

Fig. 8. Defective track located near other conductive tracks, track width is $1.5 \mathrm{~mm}$, distance to an adjacent track is $0.9 \mathrm{~mm}$, defect width is $0.1 \mathrm{~mm}$

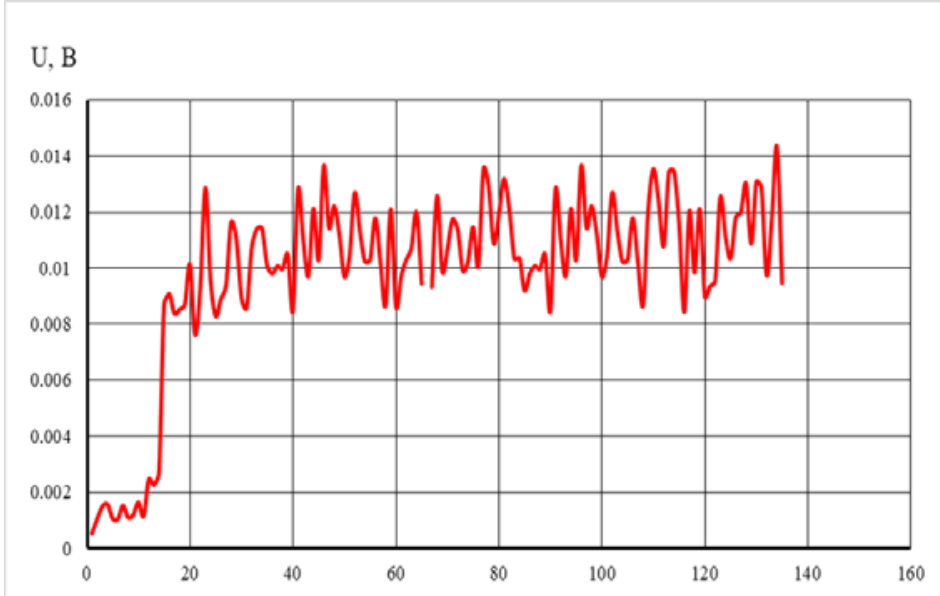

Fig. 9. Scanning results for the printed circuit board track with a defect located near another closely spaced conductive track

It is clearly visible from Fig. 9 that no significant results were obtained when scanning with one ECT, as the defect area is completely hidden behind the signal interference.

To solve this problem, the eddy-current transducer was presented in the form of two transducers with interconnected exciting and measuring windings. These transducers had the same electromagnetic characteristics and, when scanning the weld, were placed at a distance equal to or less than the width of the weld. Such arrangement of the transducers made it possible to take into account the signals from two closely spaced conductive tracks simultaneously, and subtract one signal from the other. The scanning results are shown in Fig. 10. 


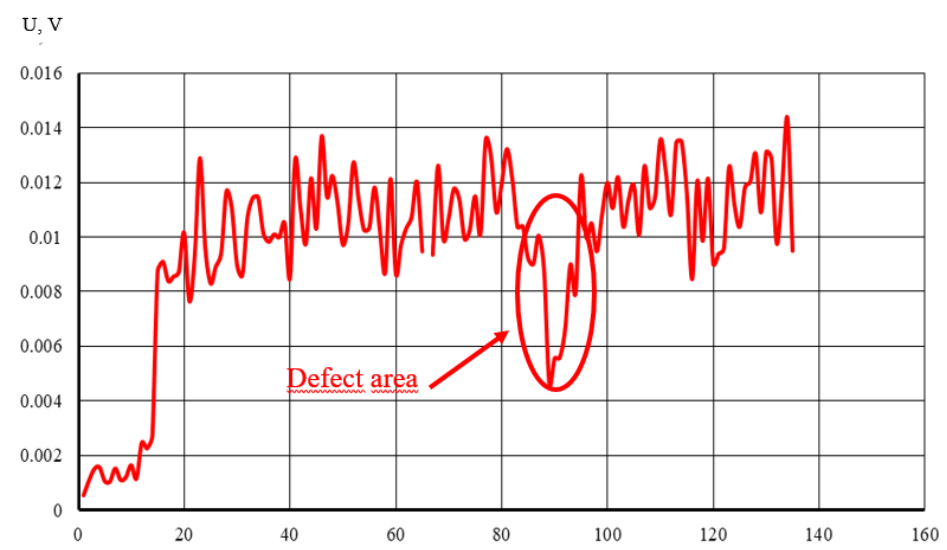

Fig. 10. Scanning results for the printed circuit board track with a defect located near another closely spaced $(0.9 \mathrm{~mm})$ conductive track using 2 ECTs

The results of the studies obtained in Fig. 10 prove convincingly the need for using two ECTs when it is necessary to scan two conductive tracks located in close proximity to each other. The defect area is localized clearly enough for its unambiguous detection.

With an even closer approach of the conductive tracks of the ECT (about $0.4 \mathrm{~mm}$ ), the localization of the defect also became difficult when using a system of two ECTs. An example of the signal received from a system of two ECTs when scanning the tracks located at a distance of $0.4 \mathrm{~mm}$ can be seen in Fig. 11.

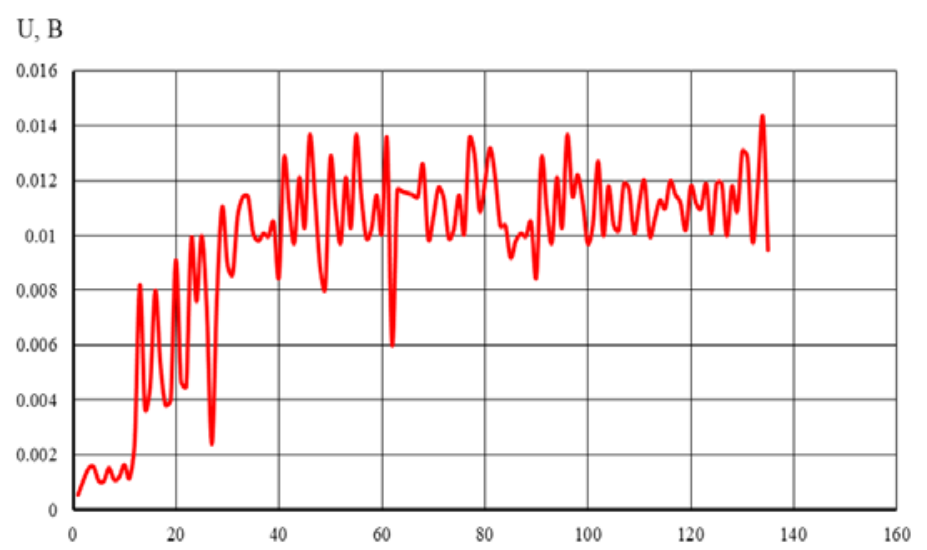

Fig. 11. Scanning results for the printed circuit board track with a defect located near another closely spaced $(0.4 \mathrm{~mm})$ conductive track using 2 ECTs

However, due to the synchronous control of the signal frequency at the exciting winding and the filtering and selective amplification system frequency, it was possible to reduce significantly the measuring winding signal noise level, which served as an informative parameter of the PCB under study. The exciting winding signal amplitude was also produced automatically, so that the complete subtraction of the informative signals from the two transducers was performed in the absence of track defects. The experimental results are shown in Fig. 12. 


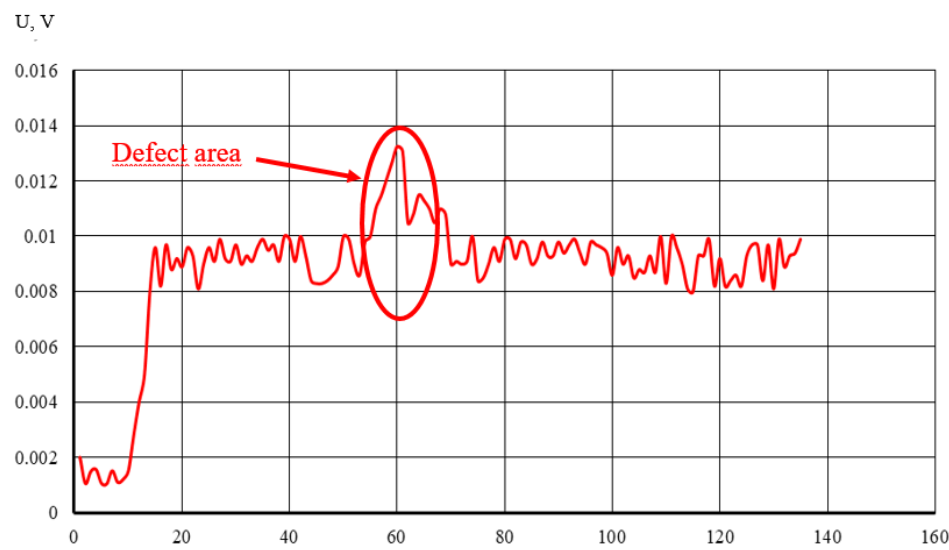

Fig. 12. Scanning results for a printed circuit board track with a defect located near a closely spaced $(0.4 \mathrm{~mm})$ conductive track using 2 ECTs and a developed filtering and selective amplification system

In is clearly visible from Fig. 12, that it became possible to identify the area of the defect due to a sharp decrease in the number of interferences (the root-mean-square deviation of the signal amplitude in the main scanning area decreased from $0.005 \mathrm{~V}$ to $0.001 \mathrm{~V}$ ) as a result of successive amplifications and filtering of the signal by various hardware and software methods. Also, the presence of a defect in this case is indicated not by a drop, but, on the contrary, by a rise in the signal amplitude. Such a phenomenon can be caused by various decompensations of the measuring and compensation windings of the used ECT, and it can be imperceptible against the interference, however, with an improvement in the quality of the receivable signal, it becomes more significant and can carry additional information about the object under control.

\section{Summary}

Thus, in the course of the conducted research, the eddy-current measuring system operating on the basis of subminiature eddy current transducers was developed with different design parameters and investigated. It was found that the material and shape of the core have a significant effect on the signal introduced into the measuring winding of the eddy-current transducer from various conductive tracks of printed circuit boards having different sizes and located at different distances from each other.

The dependences of the eddy-current transducer signal on various conducting tracks have been established. Also, an original system of filtering and selective amplification was developed making it possible to cleaning the signal received from the eddy-current transducer. The use of such a system along with the opposite connection of two eddy-current transducers made it possible to denoise significantly the received signal and detect defects located in closely spaced conductive tracks of printed circuit boards.

\section{References}

1. S. Lv, H. Kim, B. Zheng, H. Jin, A review of data mining with big data towards its applications in the electronics industry, Appl. Sci. 8(4) (2018) 1-34.

2. G. Smith, Global Consumer Electronics Manufacturing Industry-Market Research Report, Los Angeles, IBISWorld, 2019

3. M. Liukkonen, E. Havia, Y. Hiltunen, Computational intelligence in mass soldering of electronics-A survey, Expert Syst. Appl. 39 (10) (2012) 9928-9937. 
4. D. M. Tsai, Y. C. Hsieh, Machine vision-based positioning and inspection using expectation-maximization technique, IEEE Trans. Instrum. Meas. 66 (11) (2017) 28582868.

5. S. Gebus, K. Leiviskä, Knowledge acquisition for decision support systems on an electronic assembly line, Expert Syst. Appl. 36 (1) (2009) 93-101.

6. A. Ebayyeh, A. Mousavi, A Review and Analysis of Automatic Optical Inspection and Quality Monitoring Methods in Electronics Industry, IEEE Acc. 8 (2020) 183192183271.

7. I. Kovtun, J. Boiko, S. Petrashchuk, Nondestructive strength diagnostics of solder joints on printed circuit boards, Proc. 2017 Inter. Conf. Inform. Telecomm. Tech. Radio Electr. (UkrMiCo), (2017) 1-4.

8. C. D. Hechtman, In-circuit test fixture [PCB testing], IEEE Trans. Industr. Electr. 36 (2) (1989) 192-196.

9. C. Benedek, O. Krammer, M. Janoczki, L. Jakab, Solder Paste Scooping Detection by Multilevel Visual Inspection of Printed Circuit Boards, IEEE Trans. Industr. Electr. 60 (6) (2013) 2318-2331.

10. Y. C. Chan, D. J. Xie, J. K. L. Lai, I. K. Hui, Application of direct strain measurement to fatigue studies in surface solder joints, IEEE Trans. Comp. Packag. Manufactur. Tech. B. 18 (4) (1995) 715-719.

11. R. Jones, S. Galea, Health Monitoring of Composite Repairs and Joints using Optial Fibers, Comp. Struc. 50 (2002) 397-403.

12. M. Simone, T. Giorgio, G. Paolo, Structural Health Monitoring for Future Space Vehicles”, J. Intell. Mat. Sys. Struc. 17 (2006) 577-585.

13. V. S. Ramalingam, Transit Time Dependent Condition Monitoring of PCBs During Testing for Diagnostics in Electronics Industry, IEEE Trans. Industr. Electr. 65 (1) (2018) 553-560.

14. D. S. Erdahl, I. C. Ume, Online-offline laser ultrasonic quality inspection tool for multilayer ceramic capacitors-part I, IEEE Trans. Advan. Packag. 27 (4) (2004) 647-653.

15. Y. Liang, S. Zhang, A case study of the delamination analysis of plastic encapsulated microcircuits based on scanning acoustic microscope inspection, IEEE Prognos. Sys. Health Manag. Conf. (PHM-2014 Hunan) (2014) 190-193.

16. M. Reiten, R. G. Wright, Laser doppler vibrometry use in detecting faulty printed circuit boards, Proc. IEEE 2008 AUTOTESTCON (2008) 33-36.

17. S. Tismer, S. Brand, S. Klengel, M. Petzold, P. Czurratis, Acoustic imaging of bump defects in flip-chip devices using split spectrum analysis, Proc. IEEE 2013 Int. Ultrasonics Symposium (IUS) (2013) 950-953.

18. R. G. Wright, L. V. Kirkland, Circuit board testing through multispectral signal analysis, Proc. IEEE 2005 AUTOTESTCON (2005) 752-758.

19. R. G. Wright, Multiresolution sensor fusion approach to PCB fault detection and isolation, Proc. IEEE 2008 AUTOTESTCON (2008) 41-46.

20. S. Levikari, T. J. Kärkkäinen, P. Silventoinen, C. Andersson, J. Tamminen, Acoustic detection of cracks and delamination in multilayer ceramic capacitors, Proc. IEEE 2017 11th Int. Symposium Diagnostics for Electrical Machines, Power Electronics and Drives (SDEMPED) (2017) 622-627.

21. R. Shannon, G. Zucaro, J. Tallent, V. Collins, J. Carswell, A system for detecting failed electronics using acoustics, IEEE Instrument. Meas. Mag. 22 (4) (2019) 32-37. 
22. T. Dogaru, S. Smith, Giant magnetoresistance-based eddy-current sensor, IEEE Trans. Magn. 37 (2001) 3831-3838.

23. M. Cacciola, G. Megali, D. Pellicanó, F. C. Morabito, A GMR-ECT based embedded solution for applications on PCB inspections, Sens. Actuat, A 167 (2011) 25-33.

24. S. Dmitriev, V. Malikov, A. Ishkov, Superminiature eddy-current transducers for studying steel to dielectric junctions, Mater. Sci. For. 927 (2018) 161-167.

25. S. Dmitriev, V. Malikov, A. Ishkov, Measurement System for Studying Flaws in Alloy Slabs by Means of Subminiature Eddy-Current Transducers, Measur. Tech. 60(4) (2017) 372-375. 